\title{
Optimal intervention for an epidemic model under parameter uncertainty
}

\author{
Damian Clancy *, Nathan Green \\ Department of Mathematical Sciences, University of Liverpool, Liverpool, L69 7ZL, UK
}

Received 3 April 2006; received in revised form 24 August 2006; accepted 28 August 2006

Available online 7 September 2006

\begin{abstract}
We will be concerned with optimal intervention policies for a continuous-time stochastic SIR (susceptible $\rightarrow$ infective $\rightarrow$ removed) model for the spread of infection through a closed population. In previous work on such optimal policies, it is common to assume that model parameter values are known; in reality, uncertainty over parameter values exists. We shall consider the effect upon the optimal policy of changes in parameter estimates, and of explicitly taking into account parameter uncertainty via a Bayesian decisiontheoretic framework. We consider policies allowing for (i) the isolation of any number of infectives, or (ii) the immunisation of all susceptibles (total immunisation). Numerical examples are given to illustrate our results.
\end{abstract}

(C) 2006 Elsevier Inc. All rights reserved.

Keywords: Dynamic programming; Bayesian decision theory; General stochastic epidemic; Immunisation policies

\section{Introduction}

In light of recent emerging and re-emerging infections and threat of deliberate infection, the mathematical modelling of disease spread is now as important and pertinent a topic as ever. In turn, so too is the study of efficient and effective intervention strategies. One approach is via mathematical

\footnotetext{
* Corresponding author. Tel.: +44 151794 4746; fax: +44 1517944754.

E-mail address: d.clancy@liv.ac.uk (D. Clancy).
}

$0025-5564 / \$$ - see front matter (C) 2006 Elsevier Inc. All rights reserved. doi:10.1016/j.mbs.2006.08.023 
control theory. Wickwire [1] provides a thorough survey of applications of mathematical control theory to infectious disease models up to 1977; a succinct discussion of more recent work is provided by [2]. See also Chapter 7 of [3]. In the main, such work has assumed that the values of model parameters are known exactly, clearly an unrealistic assumption in practice. A notable exception in the context of continuous control (that is, when the form of intervention is to alter the rates at which certain transitions occur) is the paper [4] by Cai and Luo. Starting with an initial guess of the unknown parameter value they describe a procedure which updates this estimated value as the epidemic progresses, computing the optimal policy at any time based upon the current estimated value. They prove that an optimal policy exists and may be obtained by solving an appropriate Bellman optimality equation after each transition of the process, but do not investigate the form of the solution. In contrast to their approach, we consider a Bayesian decision-theoretic approach (see, for example, $[5,6])$, so that uncertainty about parameter values is expressed via a distribution on the set of possible values, rather than a point estimate.

Our approach also differs from that of [4] in that we consider intervention in the form of impulse control. That is, we can intervene to instantaneously change the state of the system, for instance by isolating an infective individual from the susceptible population. Such controls have been studied for the case of the general stochastic epidemic model (one of the most widely studied stochastic epidemic models, see [7], chapter 6) by Abakuks [8-10]. The intervention policies considered allowed for isolation of infectives, immunisation of susceptibles, or both. The results of Abakuks were extended to models with more general infection and removal rate functions by Clancy [11], while Kyriakidis [12,13] and Kyriakidis and Dimitrakos [14] employed a similar approach to analyse the optimal control of two competing diseases. The assumption that intervention can be effective instantaneously is of course somewhat unrealistic in practice, but mathematically a reasonable starting point.

In this paper, we consider the effect of parameter uncertainty on optimal control policies for the general stochastic epidemic model. First of all, in Section 2, we describe our model and forms of possible intervention and recall the relevant results from the literature when parameter values are supposed known. In Section 3, we consider the effect of changes in assumed parameter values, providing full proofs for the optimal isolation policy and stating corresponding results for the optimal isolation-or-total-immunisation policy. In Section 4, we show how to calculate optimal policies when parameter uncertainty is explicitly taken into account, and look at the effect of treating various different point estimates as though they were the true parameter value.

All numerical work was carried out using Matlab 6.5 on a desktop PC.

\section{The model and previously known results}

We consider the classical continuous-time SIR (susceptible $\rightarrow$ infective $\rightarrow$ removed) epidemic model known as the general stochastic epidemic model ([7] chapter 6). The population undergoing the epidemic is assumed to be of fixed size, so that there is no immigration into or emigration from the population, and to mix homogeneously. These assumptions are plausible for a small population and an epidemic of short duration. The population at time $t$ is supposed to consist of $X(t)$ susceptible, $Y(t)$ infective and $Z(t)$ removed individuals. Since the population is closed, $X(t)+Y(t)+Z(t)$ remains constant, so the process is completely described by $\{(X(t), Y(t)): t \geqslant 0\}$. 
Susceptible individuals are uninfected and vulnerable to infection; infective individuals are able to infect susceptibles (there being no latent period); removed individuals have no effect on the spread of infection having concluded their infectious period and being immune to further infection. The state of the population evolves according to a continuous-time Markov process with transition rates

$$
\left.\begin{array}{l}
P((X(t+\delta t), Y(t+\delta t))=(x-1, y+1) \mid(X(t), Y(t))=(x, y))=\beta x y \delta t+o(\delta t), \\
P((X(t+\delta t), Y(t+\delta t))=(x, y-1) \mid(X(t), Y(t))=(x, y))=\gamma y \delta t+o(\delta t),
\end{array}\right\}
$$

where $\beta>0$ is the infection rate parameter and $\gamma>0$ the removal rate parameter, and all other transitions have probability $o(\delta t)$.

In terms of the relative removal rate parameter $\rho=\gamma / \beta$, we define the embedded jump-chain transition probabilities

$$
p_{x y}=\frac{x}{x+\rho}, \quad q_{x y}=\frac{\rho}{x+\rho} .
$$

Note that for the general stochastic epidemic model $p_{x y}$ and $q_{x y}$ are independent of $y$. However, for other epidemic models this may not be the case [11].

The simplest cost function we will consider is the non-intervention expected cost function, or expected final size of the epidemic. The final size is the total number of susceptibles to be infected during the entire duration of the epidemic. Denoting by $C_{\rho}(x, y)$ the expected final size of the uncontrolled epidemic starting at $(x, y)$, then $C_{\rho}(x, y)$ is determined by

$$
\begin{aligned}
& C_{\rho}(x, y)=p_{x y}\left(1+C_{\rho}(x-1, y+1)\right)+q_{x y} C_{\rho}(x, y-1) \text { for } x, y \geqslant 1, \\
& C_{\rho}(x, 0)=0 \text { for } x \geqslant 0, C_{\rho}(0, y)=0 \text { for } y \geqslant 0 .
\end{aligned}
$$

We shall consider two forms of intervention: isolation of any number of infective individuals from the susceptible population; or the choice of either isolation of infective individuals or immunisation of the whole susceptible population. The cost of an individual becoming infected shall be fixed as the unit cost, the costs of isolation and immunisation being defined relative to the cost of infection. For the general stochastic epidemic model, Abakuks [8-10] investigated such policies assuming parameter values to be known. Abakuks [8] also considered policies allowing for the immunisation of any number of susceptibles, but was unable to obtain satisfactory analytical results due to the complicated form of the optimal policy.

Consider an impulse control policy which at any time allows for the isolation of any number of infectives at some fixed cost $L$ per individual. Defining $W_{L, \rho}(x, y)$ to be the expected future cost of waiting for a single transition to occur naturally and adopting an optimal policy from then onwards we can recursively calculate the expected future cost $V_{L, \rho}(x, y)$ of adopting an optimal policy starting at $(x, y)$ by

$$
\begin{aligned}
& W_{L, \rho}(x, y)=p_{x y}\left(1+V_{L, \rho}(x-1, y+1)\right)+q_{x y} V_{L, \rho}(x, y-1), \quad x, y \geqslant 1, \\
& V_{L, \rho}(x, y)=\min \left\{L+V_{L, \rho}(x, y-1), W_{L, \rho}(x, y)\right\}, \quad x, y \geqslant 1,
\end{aligned}
$$

with boundary conditions $V_{L, \rho}(x, 0)=0$ for $x \geqslant 0, V_{L, \rho}(0, y)=0$ for $y \geqslant 0$. In state $(x, y)$, the optimal policy is to isolate an infective if $L+V_{L, \rho}(x, y-1)<W_{L, \rho}(x, y)$ and not to intervene if 
$L+V_{L, \rho}(x, y-1)>W_{L, \rho}(x, y)$. We follow [9] in adopting the convention that if $L+V_{L, \rho}(x, y-1)$ $=W_{L, \rho}(x, y)$ then we isolate an infective. Eq. (6) is the dynamic programming equation, see, for example, equation (6.5) of [5]. Note that since there is a positive probability that at least one susceptible avoids infection, then $V_{L, \rho}(x, y) \leqslant C_{\rho}(x, y)<x$.

With this cost function Abakuks [8,9] proved that one can determine a boundary $s_{L, \rho}(x)$ such that for $1 \leqslant y \leqslant s_{L, \rho}(x)$ we intervene to isolate all the infective individuals while for $y>s_{L, \rho}(x)$ we do not intervene. The isolation boundary is given by

$$
s_{L, \rho}(x)=\max \left\{y: L+V_{L, \rho}(x, y-1) \leqslant W_{L, \rho}(x, y)\right\}=\max \left\{y: L y \leqslant W_{L, \rho}(x, y)\right\}
$$

for $x \geqslant 1$, and since the optimal isolation policy is a total isolation policy it follows that

$$
V_{L, \rho}(x, y)=\min \left\{L y, W_{L, \rho}(x, y)\right\}, \quad x, y \geqslant 1 .
$$

Abakuks [8,9] further proved that $V_{L, \rho}(x, y)$ is a non-decreasing function of each of $x, y$, and that $s_{L, \rho}(x) \leqslant s_{L, \rho}(x+1)$ for $x \geqslant 0$.

Consider now a policy which at any time allows the immunisation of either all or none of the susceptibles. Abakuks [10] proved that for this policy an immunisation boundary exists, and some associated properties. However, rather than studying the total immunisation policy alone, let us consider a policy which allows for either isolation or total immunisation. That is, at any time we may isolate any number of infective individuals each at a cost $L>0$, immunise the whole susceptible population at a cost $A+x K$, where $A \geqslant 0,0 \leqslant K<1, A+K>0$, or else do nothing. Note that for $K \geqslant 1$ the cost of non-intervention is always less than the cost of immunisation and we simply have the isolation policy as defined above. Clearly, immunisation terminates the epidemic so we will never immunise susceptibles and isolate infectives simultaneously.

Denote by $V_{L, A, K, \rho}(x, y)$ the expected future cost of adopting an optimal policy starting from $(x, y)$ and by $W_{L, A, K, \rho}(x, y)$ the expected future cost of waiting for a single transition to occur and adopting an optimal policy from then onwards. We then have

$$
\left.\begin{array}{l}
W_{L, A, K, \rho}(x, y)=p_{x y}\left(1+V_{L, A, K, \rho}(x-1, y+1)\right)+q_{x y} V_{L, A, K, \rho}(x, y-1) \text { for } x, y \geqslant 1, \\
V_{L, A, K, \rho}(x, y)=\min \left\{A+x K, L+V_{L, A, K, \rho}(x, y-1), W_{L, A, K, \rho}(x, y)\right\} \text { for } x, y \geqslant 1, \\
V_{L, A, K, \rho}(x, 0)=0 \text { for } x \geqslant 0, \quad V_{L, A, K, \rho}(0, y)=0 \text { for } y \geqslant 0,
\end{array}\right\}
$$

where $p_{x y}, q_{x y}$ are defined by (2).

In any given state $(x, y)$, if $W_{L, A, K, \rho}(x, y)<\min \left\{A+x K, L+V_{L, A, K, \rho}(x, y-1)\right\}$ we do nothing; if $L+V_{L, A, K, \rho}(x, y-1)<A+x K$ and $L+V_{L, A, K, \rho}(x, y-1) \leqslant W_{L, A, K, \rho}(x, y)$ we isolate an infective; and if $A+x K \leqslant \min \left\{L+V_{L, A, K, \rho}(x, y-1), W_{L, A, K, \rho}(x, y)\right\}$ we immunise the entire susceptible population. That is, when costs are equal, total immunisation takes precedence over isolation, which in turn takes precedence over non-intervention.

Abakuks [10] established the existence of integers $S_{L, A, K, \rho}(x)$ and $R_{L, A, K, \rho}(x)$ such that the optimal policy at $(x, y)$ isolates all infectives for $1 \leqslant y \leqslant S_{L, A, K, \rho}(x)$, takes no action for $S_{I, A, K, \rho}(x)$ $<y \leqslant R_{L, A, K, \rho}(x)$, and immunises all susceptibles for $y>R_{L, A, K, \rho}(x)$. Abakuks [10] further proved that $V_{L, A, K, \rho}(x, y)$ is a non-decreasing function of each of $x, y$, that $S_{L, A, K, \rho}(x)$ is non-decreasing in $x$, and that $S_{L, A, K, \rho}(x) \leqslant s_{L, \rho}(x)$, where $s_{L, \rho}(x)$ is the isolation boundary determined by (7). 


\section{Effect of changes in parameter values}

In this section, we examine the effects on expected costs and intervention strategies (computed under the assumption that parameter values are known) when we alter the epidemic model parameter values. In Section 3.1, an optimal isolation policy is considered; in Section 3.2 corresponding results are given when immunisation of all susceptibles is allowed as well as isolation of infectives.

\subsection{Isolation policies}

We consider first the optimal cost function for the isolation policy.

Theorem 3.1. Given any $x \geqslant 0, y \geqslant 0$, then $V_{L, \rho}(x, y)$ is a non-increasing function of $\rho$. Further, $V_{L, \rho}(x, y) \rightarrow \min \{L y, x\}$ as $\rho \rightarrow 0$ and $V_{L, \rho}(x, y) \rightarrow 0$ as $\rho \rightarrow \infty$.

Proof. We first show that $V_{L, \rho}(x, y)$ is a non-increasing function of $\rho$, by induction. The result is true for $x=0$, since $V_{L, \rho}(0, y)=0$ for $y \geqslant 0$, by definition. Suppose that for a particular $x \geqslant 1$, $V_{L, \rho}(x-1, y)$ is a non-increasing function of $\rho$ for $y \geqslant 0$. We know that for $y=0, V_{L, \rho}(x, y)=0$. Suppose that for some $y \geqslant 1, V_{L, \rho}(x, y-1)$ is a non-increasing function of $\rho$. From (5) and (2), for $\rho, \epsilon \geqslant 0$,

$$
\begin{aligned}
W_{L, \rho+\epsilon}(x, y)= & \frac{x}{\rho+\epsilon+x}\left(1+V_{L, \rho+\epsilon}(x-1, y+1)\right)+\frac{\rho+\epsilon}{\rho+\epsilon+x} V_{L, \rho+\epsilon}(x, y-1) \\
= & \frac{x}{\rho+x}\left(1+V_{L, \rho+\epsilon}(x-1, y+1)\right)+\frac{\rho}{\rho+x} V_{L, \rho+\epsilon}(x, y-1) \\
& -\frac{\epsilon x}{(\rho+\epsilon+x)(\rho+x)}\left(1+V_{L, \rho+\epsilon}(x-1, y+1)-V_{L, \rho+\epsilon}(x, y-1)\right) .
\end{aligned}
$$

By [10], Corollary 2.4, we have $1+V_{L, \rho \mid \epsilon}(x-1, y+1)-V_{L, \rho \mid \epsilon}(x, y-1) \geqslant 0$, and so

$$
W_{L, \rho+\epsilon}(x, y) \leqslant \frac{x}{\rho+x}\left(1+V_{L, \rho+\epsilon}(x-1, y+1)\right)+\frac{\rho}{\rho+x} V_{L, \rho+\epsilon}(x, y-1) .
$$

Subtracting $W_{L, \rho}(x, y)$, we obtain

$$
\begin{aligned}
W_{L, \rho+\epsilon}(x, y)-W_{L, \rho}(x, y) \leqslant & \frac{x}{\rho+x}\left(V_{L, \rho+\epsilon}(x-1, y+1)-V_{L, \rho}(x-1, y+1)\right) \\
& +\frac{\rho}{\rho+x}\left(V_{L, \rho \in \epsilon}(x, y-1)-V_{L, \rho}(x, y-1)\right) \\
\leqslant & 0
\end{aligned}
$$

by our inductive hypotheses. Then (6) gives

$$
\begin{aligned}
V_{L, \rho \mid \epsilon}(x, y) & =\min \left\{L+V_{L, \rho+\epsilon}(x, y-1), W_{L, \rho+\epsilon}(x, y)\right\} \\
& \leqslant \min \left\{L+V_{L, \rho}(x, y-1), W_{L, \rho}(x, y)\right\} \\
& =V_{L, \rho}(x, y) .
\end{aligned}
$$

So by induction on $y$ and $x, V_{L, \rho}(x, y)$ is a non-increasing function of $\rho$ for $x, y \geqslant 0$.

Next, to show that $V_{L, \rho}(x, y) \rightarrow \min \{L y, x\}$ as $\rho \rightarrow 0$, notice that the result is true for $x=0$. From (2), $p_{x y} \rightarrow 1$ and $q_{x y} \rightarrow 0$ as $\rho \rightarrow 0$. Suppose that for a particular $x \geqslant 1$, for all $y$, we have 
$V_{L, \rho}(x-1, y) \rightarrow \min \{L y, x-1\}$ as $\rho \rightarrow 0$. Then from (5), since $V_{L, \rho}(x, y-1)$ is bounded above by $x$, we have $W_{L, \rho}(x, y) \rightarrow 1+\min \{L(y+1), x-1\}$ as $\rho \rightarrow 0$. Hence $(6)$ implies that $V_{L, \rho}(x, y) \rightarrow$ $\min \{L y, L+x, L y+L+1, x\}=\min \{L y, x\}$ as $\rho \rightarrow 0$. By induction on $x$, the result follows.

Finally, to show that $V_{L, \rho}(x, y) \rightarrow 0$ as $\rho \rightarrow \infty$, notice that the result is true for $y=0$. From (2), $p_{x y} \rightarrow 0$ and $q_{x y} \rightarrow 1$ as $\rho \rightarrow \infty$. Suppose that for a particular $y \geqslant 1$, for all $x, V_{L, \rho}(x, y-1) \rightarrow 0$ as $\rho \rightarrow \infty$. Since $V_{L, \rho}(x-1, y+1)$ is bounded by $x-1$ then $(5)$ gives $W_{L, \rho}(x, y) \leqslant x$ $p_{x y}+q_{x y} V_{L, \rho}(x, y-1) \rightarrow 0$ as $\rho \rightarrow \infty$. Hence (6) implies that $V_{L, \rho}(x, y) \rightarrow 0$ as $\rho \rightarrow \infty$. By induction on $y$, the result follows.

Having looked at the effect of changes in the value of $\rho$ on the optimal cost function $V_{L, \rho}(x, y)$ we now go on to consider the effect upon the isolation boundary $s_{I, p}(x)$.

Theorem 3.2. For $x \geqslant 0$, the isolation boundary $s_{L, \rho}(x)$ is non-increasing in $\rho$. Further, $s_{L, \rho}(x) \rightarrow \max \{y \in \mathbb{Z}: y<x / L\}$ as $\rho \rightarrow 0$ and $s_{L, \rho}(x) \rightarrow 0$ as $\rho \rightarrow \infty$.

Proof. Suppose for some fixed $\rho$ that $x, y$ are such that $y \leqslant s_{L, \rho}(x)$. That is, the optimal policy requires that we isolate all of the infectives at $(x, y)$. Then $V_{L, \rho}(x, y)=L y$ and for any $\epsilon>0$ with $\epsilon \leqslant \rho$, from Theorem 3.1, $V_{I, \rho-\epsilon}(x, y) \geqslant V_{L, \rho}(x, y)=L y$. So $V_{L, \rho-\epsilon}(x, y)=L y$ which implies that $y \leqslant s_{L, \rho-\epsilon}(x)$, and it follows that $s_{L, \rho}(x)$ is non-increasing in $\rho$.

Abakuks [9] proved for $\rho>0$ that $s_{L, \rho}(x)<x / L$ for all $x$. Take $x, y \geqslant 1$ such that $y<x / L$. Then from the proof of Theorem 3.1, $W_{L, \rho}(x, y) \rightarrow \min \{L y+L+1, x\}$ as $\rho \rightarrow 0$, so that for sufficiently small $\rho>0, W_{L, \rho}(x, y)>L y$. Hence for sufficiently small $\rho>0$ we have $y \leqslant s_{L, \rho}(x)$, and it follows that $s_{L, \rho}(x) \rightarrow \max \{y \in \mathbb{Z}: y<x / L\}$ as $\rho \rightarrow 0$.

By Theorem 3.1, given $x \geqslant 0$ then $V_{L, \rho}(x, 1) \rightarrow 0$ as $\rho \rightarrow \infty$. Since the intervention cost is fixed at $L$ then $s_{L, \rho}(x)=0$ for all sufficiently large $\rho$.

Fig. 1 shows these properties for three different susceptible population sizes.

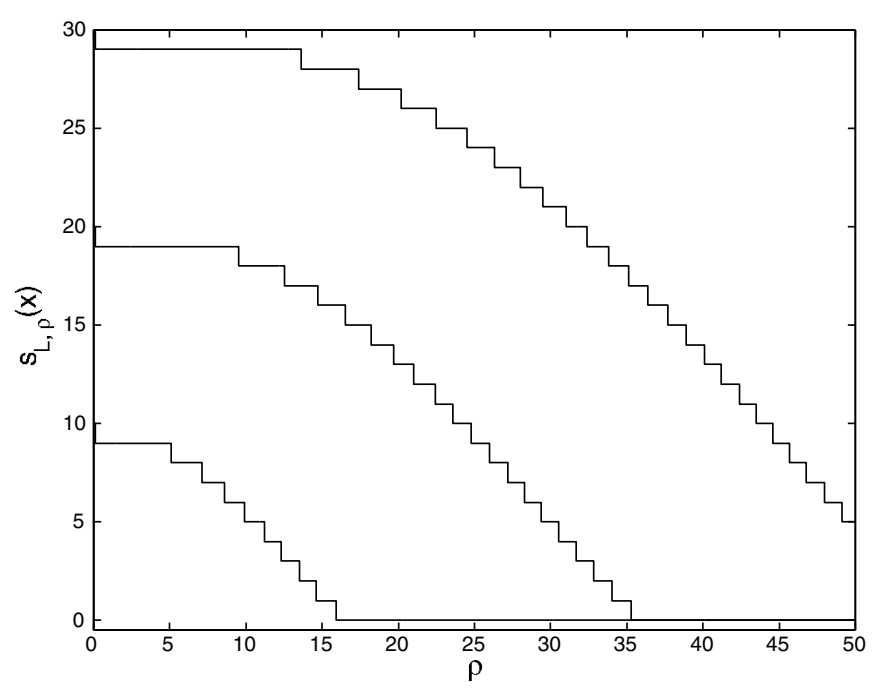

Fig. 1. The isolation boundary $s_{L, \rho}(x)$ against $\rho$ for $L=1$. Upper line represents case when $x=30$, middle line for $x=20$ and lower line for $x=10$. 
Intuitively, Theorems 3.1 and 3.2 are as one would expect. For instance, suppose that we underestimate the value of $\rho$, thereby regarding the infection as in some sense more severe than is in reality the case. Then Theorem 3.2 shows that we will intervene by isolating infectives whenever it is optimal to do so, but that we may also intervene when this is not optimal; Theorem 3.1 shows further that we will not under-estimate, but may over-estimate, the cost of the optimal policy. If we over-estimate $\rho$, converse conclusions apply.

In terms of the problem of deciding whether or not to intervene when the true value of $\rho$ is unknown, if we know the state $(x, y)$ of the population, then we intervene by isolating all infectives if $\rho<\rho_{0}(x, y)$ for some $\rho_{0}(x, y)$ with $0 \leqslant \rho_{0}(x, y) \leqslant \infty$ and otherwise do not intervene. Thus it suffices to know whether $\rho$ lies above or below $\rho_{0}(x, y)$, and it is not necessary to know the value of $\rho$ exactly. However, as the state $(x, y)$ of the process evolves, the relevant threshold value $\rho_{0}$ will also change, and the process may well pass through states $(x, y)$ such that the threshold value $\rho_{0}(x, y)$ lies within the range of plausible $\rho$ values.

Although our primary interest is in uncertainty about the epidemiological parameters $\beta, \gamma$, or equivalently uncertainty about $\rho$, it is also possible that the isolation cost $L$ will not be known exactly. Thus by analogy with the above results concerning the effect of changes in $\rho$, we have the following concerning the effect of changes in $L$.

Theorem 3.3. Given $x \geqslant 0, y \geqslant 0$, then $V_{L, \rho}(x, y)$ is a non-decreasing function of $L$. Further, $V_{L, \rho}(x, y) \rightarrow 0$ as $L \rightarrow 0$ and $V_{L, \rho}(x, y) \rightarrow C_{\rho}(x, y)$ as $L \rightarrow \infty$.

Proof. We first show that $V_{L, \rho}(x, y)$ is a non-decreasing function of $L$, by induction. The result is true for $x=0$, since $V_{L, \rho}(0, y)=0$. Suppose that for a particular $x \geqslant 1, V_{L, \rho}(x-1, y)$ is a non-decreasing function of $L$ for all $y$. We know that $V_{L, \rho}(x, 0)=0$ for $x \geqslant 0, L \geqslant 0$. Suppose now that for some $y \geqslant 1, V_{L, \rho}(x, y-1)$ is a non-decreasing function of $L$. Then

$$
\begin{aligned}
W_{L+\epsilon, \rho}(x, y)-W_{L, \rho}(x, y)= & \frac{x}{\rho+x}\left(1+V_{L+\epsilon, \rho}(x-1, y+1)\right)+\frac{\rho}{\rho+x} V_{L+\epsilon, \rho}(x, y-1) \\
& -\frac{x}{\rho+x}\left(1+V_{L, \rho}(x-1, y+1)\right)-\frac{\rho}{\rho+x} V_{L, \rho}(x, y-1) \\
\geqslant & 0,
\end{aligned}
$$

by our inductive hypotheses. Thus

$$
\begin{aligned}
V_{L+\epsilon, \rho}(x, y) & =\min \left\{L+\epsilon+V_{L+\epsilon, \rho}(x, y-1), W_{L+\epsilon, \rho}(x, y)\right\} \\
& \geqslant \min \left\{L+V_{L, \rho}(x, y-1), W_{L, \rho}(x, y)\right\} \\
& =V_{L, \rho}(x, y) .
\end{aligned}
$$

So by induction on $y$ and $x, V_{L, \rho}(x, y)$ is a non-decreasing function of $L$ for $x, y \geqslant 0$.

Next, since $V_{L, \rho}(x, y)=\min \left\{L y, W_{L, \rho}(x, y)\right\}$ it is clear that $V_{L, \rho}(x, y) \rightarrow 0$ as $L \rightarrow 0$ for any $x, y \geqslant 0$.

Finally, to show that $V_{L, \rho}(x, y) \rightarrow C_{\rho}(x, y)$ as $L \rightarrow \infty$, note that for $L>x$ the cost of isolating a single infective is greater than the cost due to the infection of all susceptibles, so that it is optimal never to isolate any infectives and hence $V_{L, \rho}(x, y)=C_{\rho}(x, y)$ for $L>x$. 
We conjecture that the isolation boundary $s_{L, \rho}(x)$ is non-increasing in $L$ for all $x \geqslant 0, L \geqslant 0$ and numerical evidence supports this. We have only been able to obtain partial results (see [15]); however, we do have the following.

Theorem 3.4. Given $x \geqslant 1$, then $s_{L, \rho}(x) \rightarrow \infty$ as $L \rightarrow 0$ and $s_{L, \rho}(x) \rightarrow 0$ as $L \rightarrow \infty$.

Proof. Fix $x, y \geqslant 1$. From (5), $W_{L, \rho}(x, y) \geqslant p_{x y}=x /(x+\rho)>0$, so that for sufficiently small $L$ we have $L y<W_{L, \rho}(x, y)$ and the optimal policy at $(x, y)$ is to isolate all infectives. That is, for any $x, y \geqslant 1$ then $s_{L, \rho}(x) \geqslant y$ for all sufficiently small $L$, so that $s_{L, \rho}(x) \rightarrow \infty$ as $L \rightarrow 0$.

From Theorem 3.3, for $x \geqslant 1, V_{L, \rho}(x, 1) \rightarrow C_{\rho}(x, 1)$ as $L \rightarrow \infty$ which implies that for sufficiently large $L, V_{L, \rho}(x, 1)<L$, so that $s_{L, \rho}(x)=0$ for all sufficiently large $L$.

Fig. 2 shows examples of these properties for three different susceptible population sizes.

\subsection{Isolation or total immunisation policies}

In this section, we shall state without proof results corresponding to those of Section 3.1 for the isolation-or-total-immunisation policy. The proofs are given in [15] and are straightforward modifications of the proofs given for the optimal isolation policy in Section 3.1.

Theorem 3.5. Given $x \geqslant 0, y \geqslant 0$, then the optimal cost function $V_{L, A, K, \rho}(x, y)$ defined by Eq. (8) satisfies

(i) $V_{L, A, K, \rho}(x, y)$ is a non-increasing function of $\rho$ with $V_{L, A, K, \rho}(x, y) \rightarrow \min \{L y, A+x K, x\}$ as $\rho \rightarrow 0$ and $V_{L, A, K, \rho}(x, y) \rightarrow 0$ as $\rho \rightarrow \infty$;

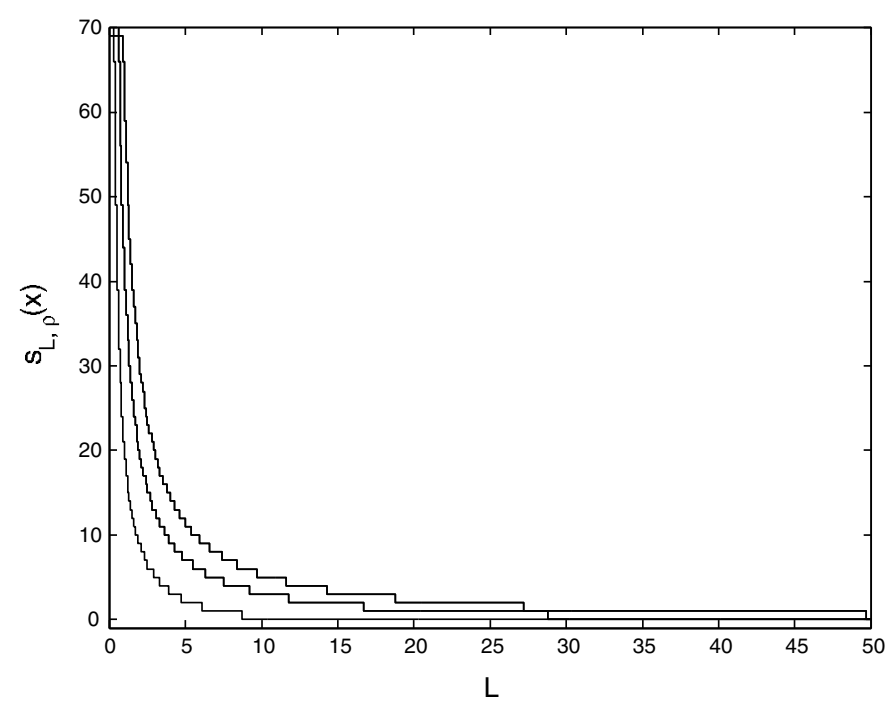

Fig. 2. The isolation boundary $s_{L, \rho}(x)$ against $L$ for $\rho=5$. Upper line represents case when $x=30$, middle line for $x=20$ and lower line for $x=10$. 
(ii) $V_{L, A, K, \rho}(x, y)$ is a non-decreasing function of each of $L, A$ and $K$ with $V_{L, A, K, \rho}(x, y) \rightarrow 0$ as $\min \{L, A+K\} \rightarrow 0$, and $V_{L, A, K, \rho}(x, y) \rightarrow C_{\rho}(x, y)$ as $\min \{L, A+K\} \rightarrow \infty$.

Recall the form of the optimal isolation-or-total-immunisation policy: there exist integers $S_{L, A, K, \rho}(x)$ and $R_{L, A, K, \rho}(x)$ such that the optimal policy at $(x, y)$ isolates all infectives for $1 \leqslant y \leqslant S_{L, A, K, \rho}(x)$, takes no action for $S_{L, A, K, \rho}(x)<y \leqslant R_{L, A, K, \rho}(x)$, and immunises all susceptibles for $y>R_{L, A, K, \rho}(x)$. Abakuks [10] proved that $S_{L, A, K, \rho}(x)=R_{L, A, K, \rho}(x)$ for all large enough $x$, and that defining

$$
\phi(x)=\max \{y \in \mathbb{Z}: L y<A+x K\} \text { for } x \geqslant 1
$$

then if $S_{L, A, K, \rho}(x)=R_{L, A, K, \rho}(x)$ it follows that $S_{L, A, K, \rho}(x)=\phi(x)=R_{L, A, K, \rho}(x)$. Note that for any $x \geqslant 1, \phi(x)$ is non-increasing in $L$ and non-decreasing in each of $A, K$.

Similarly to Theorem 3.2 for the optimal isolation boundary, we have the following.

Theorem 3.6. Given $x \geqslant 0$,

(i) $S_{L, A, K, \rho}(x)$ is non-increasing in $\rho$;

(ii) $R_{L, A, K, \rho}(x)$ is non-decreasing in $\rho$;

(iii) For $x<A /(1-K)$ then $S_{L, A, K, \rho}(x) \rightarrow \max \{y \in \mathbb{Z}: y<x / L\}$ and $R_{L, A, K, \rho}(x) \rightarrow \infty$ as $\rho \rightarrow 0$; for $x \geqslant A /(1-K)$ then $S_{L, A, K, \rho}(x) \rightarrow \phi(x)$ and $R_{L, A, K, \rho}(x) \rightarrow \phi(x)$ as $\rho \rightarrow 0$.

(iv) $S_{L, A, K, \rho}(x) \rightarrow 0$ and $R_{L, A, K, \rho}(x) \rightarrow \infty$ as $\rho \rightarrow \infty$.

In summary, we have investigated the effect of changes in parameter values on the optimal cost function and on the optimal action, firstly for an isolation policy and subsequently for an isolation-or-total-immunisation policy. Our results show that if the state $(x, y)$ of the process is known, then to determine the optimal action it is not necessary to know the precise value of $\rho$, but only to know whether $\rho$ lies above or below certain threshold values $\rho_{0}(x, y)$. However, the relevant threshold values change as the state $(x, y)$ of the process evolves; furthermore, it will generally be more realistic to represent our knowledge of $\rho$ by some uncertainty distribution, rather than trying to determine with certainty whether $\rho$ lies above or below certain threshold values. This is the situation which we consider in the following section.

\section{Distributional representation of parameter uncertainty}

In this section, we explore the use of an uncertainty distribution to represent our incomplete knowledge of parameter values. We adopt a Bayesian decision-theoretic approach to determine the optimal policy (see, for example, $[5,6]$ ). In a full Bayesian analysis, we would assign a prior distribution to each unknown parameter, which is then updated in the light of the observed progress of the epidemic process to give a posterior distribution at any time $t>0$. The expected future cost of an optimal policy is then computed using this posterior distribution, and taking into account the fact that the posterior distribution will be further updated in the future. However, this leads to a rather complicated optimisation problem, due to the need to take into account the future updating of the posterior distribution in addition to the transitions of the process $(x, y)$ itself. 
For a rigorous analysis of such problems in the context of discrete time processes, see [16]. To avoid such an overly involved analysis, we will take the more straightforward approach of assigning an uncertainty distribution to our unknown parameter but not updating this distribution at all as the epidemic progresses. This seems reasonable in practice as our knowledge of parameter values at the start of an epidemic may well be based on more than one completed previous epidemics, in which case the further information provided by the epidemic in progress will be of relatively little value. Note that although we adopt a Bayesian decision theoretic approach, we do not explicitly carry out any Bayesian inference.

\subsection{Optimal intervention under parameter uncertainty}

As before, we consider first the optimal isolation policy, before moving on to the isolation-ortotal-immunisation policy. Suppose our knowledge about the relative removal rate $\rho$ is represented by some distribution $\pi$ on $0 \leqslant \rho<\infty$. Denote by $V_{L}^{\pi}(x, y)$ the expected future cost of adopting an optimal isolation policy starting from $(x, y)$ and by $W_{L}^{\pi}(x, y)$ the expected future cost of waiting for a single transition to occur and adopting an optimal policy from then onwards. Note that $V_{L}^{\pi}(x, y)$ and $W_{L}^{\pi}(x, y)$ each represent expectations with respect to both the uncertainty distribution $\pi$ and the (random) future progress of the infection process, under the optimal (non-random) intervention policy. Denote by $V_{L}^{\pi}(x, y \mid \rho)$ the expected future cost, starting from $(x, y)$, of adopting the policy which is optimal under $\pi$, conditional upon the true value of the relative removal rate parameter being $\rho$. Then $V_{L}^{\pi}(x, y)=\mathbb{E}_{\pi}\left[V_{L}^{\pi}(x, y \mid \rho)\right]$, and noting that the transition probabilities $p_{x y}$, $q_{x y}$ given by (2) are themselves functions of $\rho$ then corresponding to (5) and (6) we have

$$
\begin{aligned}
& W_{L}^{\pi}(x, y)=\mathbb{E}_{\pi}\left[p_{x y}\left(1+V_{L}^{\pi}(x-1, y+1 \mid \rho)\right)+q_{x y} V_{L}^{\pi}(x, y-1 \mid \rho)\right], \\
& V_{L}^{\pi}(x, y)=\min \left\{L+V_{L}^{\pi}(x, y-1), W_{L}^{\pi}(x, y)\right\} .
\end{aligned}
$$

In state $(x, y)$ we intervene by isolating an infective whenever $L+V_{L}^{\pi}(x, y-1) \leqslant W_{L}^{\pi}(x, y)$. It follows that

$$
\begin{aligned}
& V_{L}^{\pi}(x, y \mid \rho) \\
& = \begin{cases}L+V_{L}^{\pi}(x, y-1 \mid \rho) & \text { if } L+V_{L}^{\pi}(x, y-1) \leqslant W_{L}^{\pi}(x, y), \\
p_{x y}\left(1+V_{L}^{\pi}(x-1, y+1 \mid \rho)\right)+q_{x y} V_{L}^{\pi}(x, y-1 \mid \rho) & \text { otherwise. }\end{cases}
\end{aligned}
$$

Using Eqs. (10)-(12), the optimal policy can be evaluated numerically as follows. Assuming that $\pi$ is a continuous distribution, then for some (sufficiently large) positive integer $n$, denote by $\rho_{0}, \rho_{1}, \ldots, \rho_{n}$ the quantiles of the distribution $\pi$ such that for $i=0,1, \ldots, n, \pi\left(\rho \leqslant \rho_{i}\right)=(i+0.5) /$ $(n+1)$. Fix $y_{\max } \geqslant 1$. Working through states in the order $(1,1),(1,2), \ldots,\left(1, y_{\max }\right)$, $(2,1),(2,2), \ldots,\left(2, y_{\max }-1\right), \ldots$, then when we reach state $(x, y)$ we will already have dealt with the states $(x-1, y+1)$ and $(x, y-1)$, so that we know the values of $V_{L}^{\pi}\left(x-1, y+1 \mid \rho_{i}\right)$ and $V_{L}^{\pi}\left(x, y-1 \mid \rho_{i}\right)$ for $i=0,1, \ldots, n$, together with the optimal action in each of these two states. The expectation on the right hand side of (10) can now be approximated as

$$
W_{L}^{\pi}(x, y) \approx \frac{1}{n} \sum_{i=0}^{n} p_{x y}\left(\rho_{i}\right)\left(1+V_{L}^{\pi}\left(x-1, y+1 \mid \rho_{i}\right)\right)+q_{x y}\left(\rho_{i}\right) V_{L}^{\pi}\left(x, y-1 \mid \rho_{i}\right) .
$$


Substituting the above approximation into Eq. (11) allows us to determine the optimal action in state $(x, y)$, together with the associated $\operatorname{cost} V_{L}^{\pi}(x, y)$. Finally, using Eq. (12) we can compute $V_{L}^{\pi}\left(x, y \mid \rho_{i}\right)$ for $i=0,1, \ldots, n$. To choose an appropriate value for $n$ in practice, we can take an increasing set of $n$ values and compute the (approximate) optimal policy by the above method for each $n$ in turn, stopping when further increases in $n$ do not result in any changes to the computed optimal action within the region of states $(x, y)$ under consideration.

The optimal policy under an uncertainty distribution $\pi$ unfortunately does not in general have the simple form elaborated in Section 2 for the case when the true value of $\rho$ is known. For example, Fig. 3(i) shows the optimal isolation policy obtained when we take $\pi$ to be the Exponential distribution with mean 100 , with $L=0.5$. We see that the optimal policy in this case is not a stopping rule. For the computation we took $n=999$, so that 1000 quantiles were used to approximate the Exponential distribution. The computed policy is identical to that found with $n=899$, so it at least seems plausible to suppose that the approximation method has converged to the true solution. Whatever finite $n$ value we use, we can never be certain that the computed policy is exactly correct for $\pi$ the Exponential distribution with mean 100; however, our method is exact (subject to machine accuracy) if instead we take $\pi$ to be the discrete uniform distribution on the set of quantiles $\left\{\rho_{0}, \rho_{1}, \ldots, \rho_{999}\right\}$ of the Exponential distribution with mean 100. Thus, we have certainly demonstrated the existence of an uncertainty distribution $\pi$ such that the optimal isolation policy takes the complicated form shown in Fig. 3(i). We could simplify the problem by looking for the optimal total isolation policy, in the same way as in Section 2 we considered the optimal total immunisation policy. That is, in any state $(x, y)$ we choose whether to isolate the whole infective population, or not to intervene. However, we still find that the set of states $(x, y)$ in which it is optimal to intervene can be quite complicated, and in particular need not be a connected region of the $(x, y)$ plane.

(i)

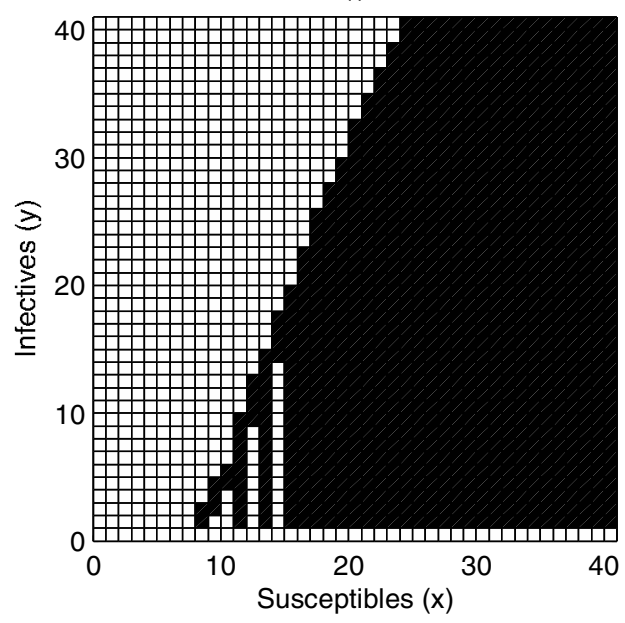

(ii)

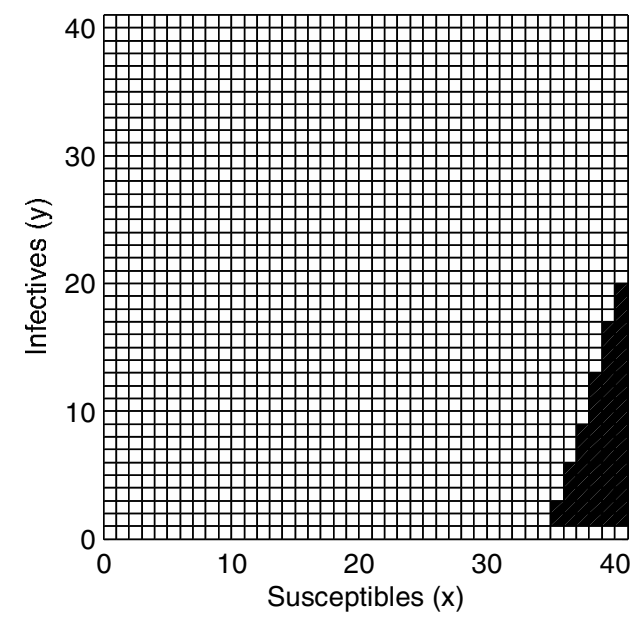

Fig. 3. Optimal isolation regions, illustrating the effect of replacing the unknown parameter $\rho$ by the point estimate $\bar{\rho}$. Black area indicates states $(x, y)$ where it is optimal to isolate an infective; white area indicates states where it is optimal not to intervene. In each case, the cost of isolating an infective is taken to be $L=0.5$. (i) Uncertainty about $\rho$ is represented by taking $\pi$ to be the Exponential distribution with mean 100. (ii) $\rho$ is assumed to take the value $\bar{\rho}=100$. 
The complicated form of the isolation region, as illustrated in Fig. 3(i), means that, aside from difficulties of mathematical analysis, the optimal policy under $\pi$ may not be easy to implement in practice. In previous work on optimal control of epidemic processes, it is usual to treat parameter values as known; in reality, this means that a point estimate must be used. Regarding this point estimate as the true parameter value means that the inherent uncertainty in the estimate is ignored, but does result in a simpler form of optimal policy, and may therefore be preferred. The obvious choice is to use the point estimate $\bar{\rho}=\mathbb{E}_{\pi}[\rho]$ and compute the optimal policy under the assumption that $\rho$ is known to take the value $\bar{\rho}$. For example, corresponding to Fig. 3(i) we have $\mathbb{E}_{\pi}[\rho]=100$. Fig. 3(ii) shows the optimal isolation policy obtained if we assume that $\rho$ is known and takes the value $\rho=\bar{\rho}=100$, with $L=0.5$. Comparing Fig. 3(ii) with Fig. 3(i) we see that treating $\rho$ as known, with value $\bar{\rho}$, does not necessarily produce a very good approximation to the true optimal policy under $\pi$. However, $\bar{\rho}$ is not the only possible choice of point estimate for $\rho$. In particular, we will now consider some alternative parameterisations of the general stochastic epidemic model which give rise to alternative natural estimates for $\rho$.

\subsection{Alternative model parameterisations}

So far, we have parameterised our model in terms of the relative removal rate $\rho$. Two possible alternatives often used in the literature are to parameterise in terms of either (i) the basic reproduction number $R_{0}$, defined to be the average number of new infections directly attributable to a single infective in an otherwise wholly susceptible population; or (ii) the avoidance probability $q$, defined to be the probability that a single infective fails to infect one specific susceptible individual. The basic reproduction number $R_{0}$ is usually used as a threshold parameter for an epidemic model: for a large population, the epidemic never takes off if $R_{0} \leqslant 1$ and takes off with non-zero probability if $R_{0}>1$. Intervention to reduce $R_{0}$ to below 1 is thus a common epidemic control strategy (see, for example, [17] or [18]).

The relationships between the parameters $\rho, R_{0}, q$ are as follows. Firstly, since the average length of an infectious period is $1 / \gamma$ and the number of infections per unit time in a wholly susceptible population of size $N$ is $\beta N$, then

$$
R_{0}=\beta N / \gamma=N / \rho
$$

Denoting by $I$ the length of a typical infectious period, so that $I \sim \operatorname{Exp}(\gamma)$, the probability that one particular susceptible escapes infection from a single infective is

$$
q=\mathbb{E}\left[\mathrm{e}^{-\beta I}\right]=\frac{\gamma}{\gamma+\beta}=\frac{1}{1+(1 / \rho)} .
$$

Note in particular that Eqs. (13) and (14) imply that the parameters $\rho, R_{0}, q$ are monotonically related: an increase in $\rho$ corresponds to a decrease in $R_{0}$ and to an increase in $q$. Thus the results of Section 3 can be immediately re-stated in terms of $R_{0}$ or $q$. For instance, given $x \geqslant 0, y \geqslant 0$, then $V_{L, \rho}(x, y)$ is non-decreasing in $R_{0}$ and non-increasing in $q$, and the isolation boundary $s_{L, \rho}(x)$ is non-decreasing in $R_{0}$ and non-increasing in $q$.

Since the parameter $R_{0}$ involves population size $N$, whereas $\rho$ and $q$ do not, we shall define $R=1 / \rho$ and consider $R$ rather than $R_{0}$. Inverting relationship (14) we then have 


$$
\rho=\frac{1}{R}=\frac{q}{1-q} .
$$

In approximating the optimal policy under $\pi$ by the optimal policy when $\rho$ is known to take some particular value, the relationships (15) lead to the following three natural choices of point estimate for $\rho$.

$$
\text { (i) } \bar{\rho}=\mathbb{E}_{\pi}[\rho] ; \quad \text { (ii) } \rho_{q}=\frac{\mathbb{E}_{\pi}[q]}{1-\mathbb{E}_{\pi}[q]} ; \quad \text { (iii) } \rho_{R}=\frac{1}{\mathbb{E}_{\pi}[R]} .
$$

We will now investigate the effect of using the above point estimates of $\rho$ upon the computed optimal intervention policy and associated cost function.

\subsection{Effect of different parameter estimates on the optimal policy}

For the estimates $\bar{\rho}, \rho_{q}, \rho_{R}$, it turns out that there is a particularly simple relationship between the three optimal isolation policies corresponding to these three different estimates for $\rho$, as shown by the following result.

Theorem 4.1. Provided $\mathbb{E}_{\pi}[\rho], \mathbb{E}_{\pi}[R]<\infty$ then

(i) For any $x, y \geqslant 0, V_{L, \bar{\rho}}(x, y) \leqslant V_{L, \rho_{q}}(x, y) \leqslant V_{L, \rho_{R}}(x, y)$;

(ii) For any $x \geqslant 0, s_{L, \bar{\rho}}(x) \leqslant s_{L, \rho_{q}}(x) \leqslant s_{L, \rho_{R}}(x)$.

Proof. From (15), we see that $\rho$ is a convex function of $q$, so that by Jensen's inequality $\bar{\rho} \geqslant \rho_{q}$. Similary from (15) we have $R=(1-q) / q$, so that $R$ is a convex function of $q$, $\mathbb{E}_{\pi}[R] \geqslant\left(1-\mathbb{E}_{\pi}[q]\right) / \mathbb{E}_{\pi}[q]$ and hence $\rho_{q} \geqslant \rho_{R}$. Applying Theorems 3.1 and 3.2, the results follow.

Fig. 4 illustrates the effect upon the isolation region of using each of the three estimates $\bar{\rho}, \rho_{q}$, $\rho_{R}$. Whereas in computing Fig. 3 we used a standard (Exponential) distribution for $\rho$, we now parameterise initially in terms of the avoidance probability $q$, taking $q$ to follow the Beta distribution with parameters $(5,0.25)$ (so that $E_{\pi}[q]=5 / 5.25=0.952$ ). The induced distribution $\pi$ of $\rho$ is therefore non-standard, but this does not cause any problems in practice. The cost of isolating an infective is taken to be $L=0.7$. Fig. 4(i) shows the isolation region computed under the uncertainty distribution $\pi$, which is seen to have a rather complicated form similar to that seen previously in Fig. 3(i). As for Fig. 3(i) the optimal policy was computed with $n=999$, the computed policy being identical to that found with $n=899$ so that it is plausible to suppose convergence has been achieved. Figs. 4(ii)-(iv) show the isolation regions computed by treating the point estimates $\bar{\rho}, \rho_{q}, \rho_{R}$, respectively, as though they were the true values of $\rho$. These figures illustrate the relationships between the boundaries $s_{L, \bar{\rho}}, s_{L, \rho_{q}}, s_{L, \rho_{R}}$ expressed in Theorem 4.1 (ii). We also see that in this case, as in Fig. 3, using $\bar{\rho}$ provides a poor approximation to the true isolation region. On the other hand, using either of $\rho_{q}, \rho_{R}$ yields an isolation region which is firstly a good approximation to the true isolation region, and secondly of simpler form than the true isolation region and hence more appropriate for practical implementation. 
(i)

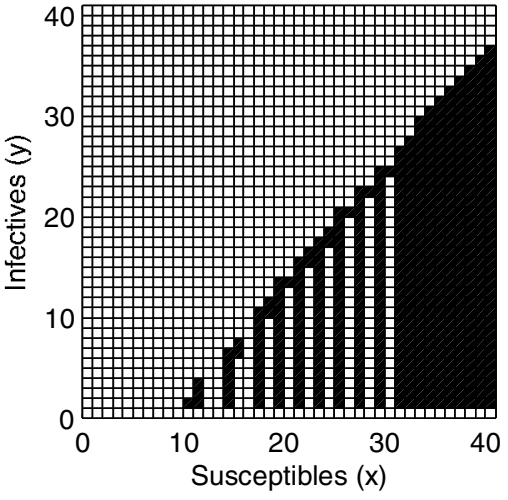

(iii)

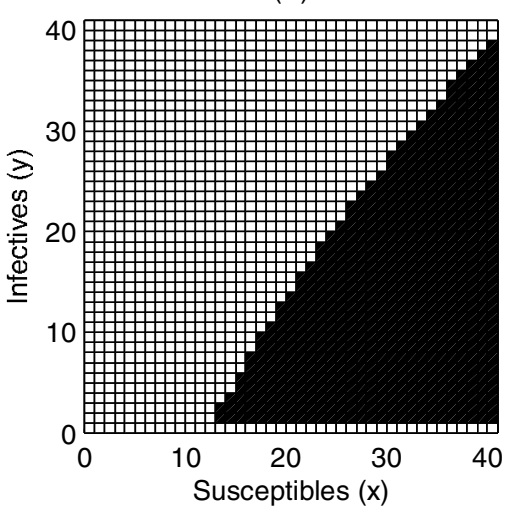

(ii)

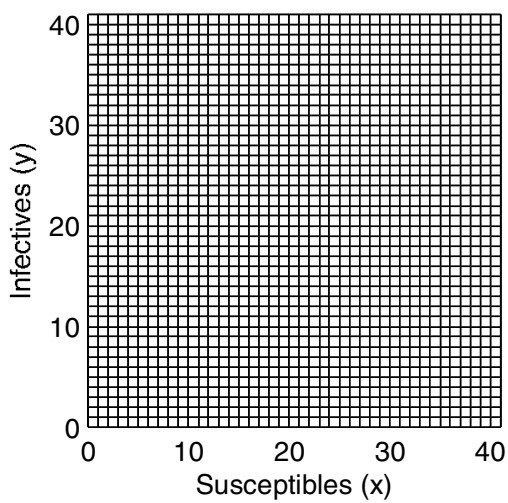

(iv)

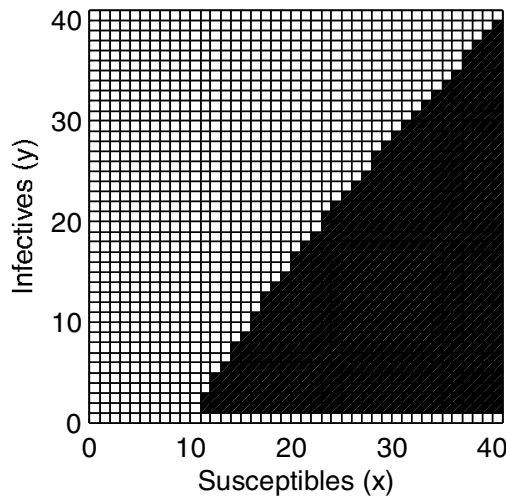

Fig. 4. Optimal isolation regions, illustrating the effect of replacing the unknown parameter $\rho$ by each of the point estimates $\bar{\rho}, \rho_{q}$ or $\rho_{R}$. Black area indicates states $(x, y)$ where it is optimal to isolate an infective; white area indicates states where it is optimal not to intervene. In each case, the cost of isolating an infective is taken to be $L=0.7$. (i) Uncertainty about parameter values is represented by taking $q$ to follow the Beta distribution with parameters $(5,0.25)$, so $\mathbb{E}_{\pi}[q]=5 / 5.25=0.952$. (ii) $\rho$ is assumed to take the value $\bar{\rho}=4.46 \times 10^{9}$. (iii) $\rho$ is assumed to take the value $\rho_{q}=20$. (iv) $\rho$ is assumed to take the value $\rho_{R}=16.07$.

For the isolation-or-total-immunisation policy we have the following results corresponding to Theorem 4.1.

Theorem 4.2. Provided $\mathbb{E}_{\pi}[\rho], \mathbb{E}_{\pi}[R]<\infty$ then

(i) For any $x, y \geqslant 0, V_{L, A, K, \bar{\rho}}(x, y) \leqslant V_{L, A, K, \rho_{q}}(x, y) \leqslant V_{L, A, K, \rho_{R}}(x, y)$;

(ii) For any $x \geqslant 0, S_{L, A, K, \bar{\rho}}(x) \leqslant S_{L, A, K \rho_{q}}(x) \leqslant S_{L, A, K, \rho_{R}}(x) \leqslant R_{L, A, K, \rho_{R}} \leqslant R_{L, A, K, \rho_{q}} \leqslant R_{L, A, K, \bar{\rho}}$.

Proof. From the proof of Theorem 4.1 we know that $\bar{\rho} \geqslant \rho_{q} \geqslant \rho_{R}$, and hence the results follow from Theorems 3.5 and 3.6. 
Fig. 5 illustrates the effect upon the computed isolation-or-total-immunisation policy of using each of the three estimates $\bar{\rho}, \rho_{q}, \rho_{R}$. In this case, we parameterise initially in terms of $R$, taking $R$ to follow the Exponential distribution with mean 0.05. We take cost parameters $L=1.5, A=0$, $K=0.5$. Fig. 5(i) shows the optimal policy computed under the appropriate uncertainty distribution, where as before we took $n=999$ for the computation and this gave identical results to taking $n=899$. Figs. 5(ii)-(iv) show the policies computed by treating the point estimates $\bar{\rho}, \rho_{q}, \rho_{R}$, respectively, as though they were the true values of $\rho$. Once again, whereas using $\bar{\rho}$ provides a poor approximation to the true optimal policy, using either of $\rho_{q}, \rho_{R}$ yields a reasonably good approximation.

It is important to note that Figs. 3-5 are intended to be illustrative of problems which may arise when uncertainty in parameter values is ignored, and not to represent typical situations. If the le-

(i)

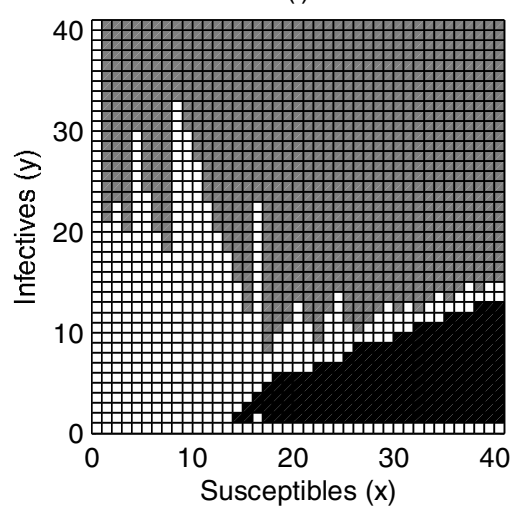

(iii)

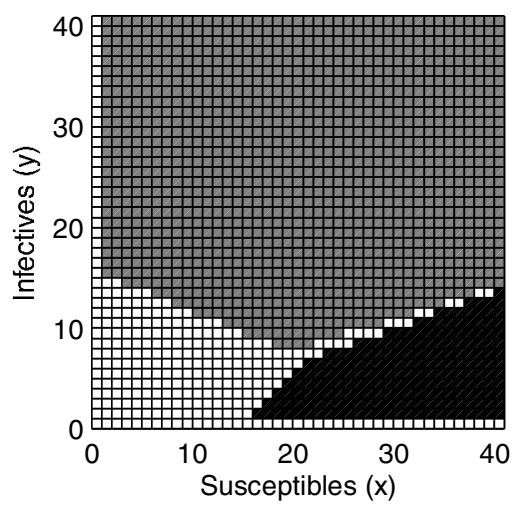

(ii)

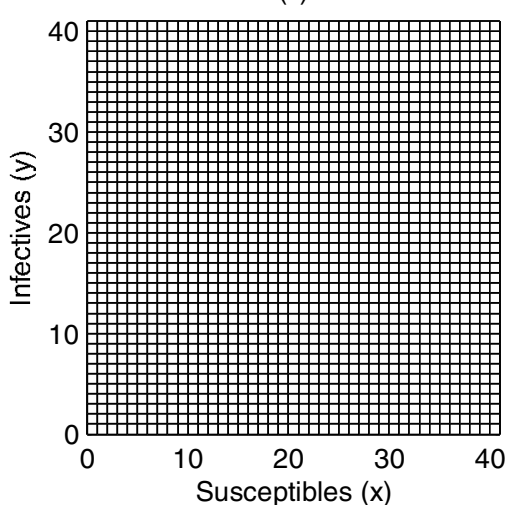

(iv)

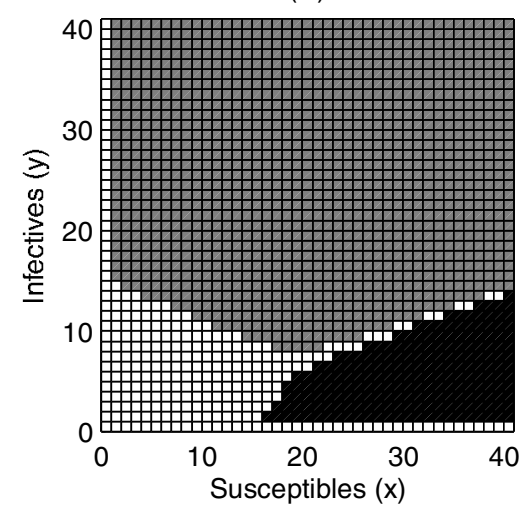

Fig. 5. Optimal isolation-or-total-immunisation policies, illustrating the effect of replacing the unknown parameter $\rho$ by each of the point estimates $\bar{\rho}, \rho_{q}$ or $\rho_{R}$. Black area indicates states $(x, y)$ where it is optimal to isolate an infective; grey area indicates states where total immunisation is optimal; white area indicates states where it is optimal not to intervene. In each case, $L=1.5, A=0, K=0.5$. (i) Uncertainty about parameter values is represented by taking $R$ to follow the Exponential distribution with mean 0.05. (ii) $\rho$ is assumed to take the value $\bar{\rho}=165.88$. (iii) $\rho$ is assumed to take the value $\rho_{q}=20.92$. (iv) $\rho$ is assumed to take the value $\rho_{R}=20$. 
vel of uncertainty is low, so that $\pi$ is a highly informative (low variance) distribution, then using any of the estimates $\bar{\rho}, \rho_{q}, \rho_{R}$ will result in a good approximation to the true optimal policy. However, when there is a high level of uncertainty about parameter values we have seen that the choice of estimate can be important. For the examples shown in Figs. 4 and 5, both $\rho_{q}$ and $\rho_{R}$ perform well, whereas $\bar{\rho}$ performs poorly. Of course, $\bar{\rho}, \rho_{q}, \rho_{R}$ are not the only possible estimates which could be used, but the results of this section do demonstrate the importance of choice of parameter estimate if uncertainty is to be ignored.

\section{Discussion}

In this paper, we have investigated optimal intervention for a particular stochastic epidemic model when faced with parameter uncertainty. In Section 3, we established results to the effect that, as one would expect, a more severe infection calls for a greater level of intervention. In Section 4, we looked at the form of the optimal policy when parameter uncertainty is explicitly taken into account, and at the effect upon the computed policy of treating three different point estimates as the true parameter value. We saw that choice of parameter estimate can have a substantial effect upon the computed intervention policy.

A key remaining problem is to elaborate conditions under which use of any specific parameter estimate leads to good approximation of the true optimal intervention policy. From Theorems 4.1 and 4.2 we know that using the estimate $\rho_{q}$ results in a policy intermediate between those computed under $\bar{\rho}$ or under $\rho_{R}$, so that $\rho_{q}$ might be favoured as a reasonable compromise. This is to some extent supported by Figs. 4 and 5, which show the use of $\rho_{q}$ leading to a good approximation to the true optimal policy in each case. Further practical (minor) advantages of $\rho_{q}$ are: (i) that we need only deal with an uncertainty distribution on the finite closed interval $[0,1]$, whereas parameterising in terms of either $\rho$ or $R$ requires an uncertainty distribution on $[0, \infty$ ); (ii) that we can reasonably represent a lack of prior information by taking $q$ to be uniformly distributed on $[0,1]$; (iii) that a flexible family of distributions on $[0,1]$ are available in the form of the Beta distributions. However, the real question is whether using $\rho_{q}$ leads to good approximation of the true optimal policy in any particular case, and this can as yet only be answered by actually computing the true optimal policy.

It is important to be aware that our results apply to one specific epidemic model, allowing for certain rather specific interventions, and assuming a particular simple cost function. Further work to investigate other models, other forms of intervention, and other cost functions would thus be very valuable. Various alternative forms of intervention and cost functions are described in the review papers $[1,2]$. One way in which we could extend the model would be to allow more general transition rate functions. Clancy [2] examined optimal isolation and total immunisation policies for a model with quite general infection and removal rate functions. In particular, a common modification, suggested by Severo [19], is to replace the constant parameters $\beta, \gamma$ by state-dependent functions $\beta_{x y}=\beta x^{-h} y^{a-1}, \gamma_{x y}=\gamma y^{c}$ for $\beta, \gamma, a, b, c$ constant. Severo refered to $a, b, c$ as the infection power, the safety-in-numbers power and the removal power, respectively, and proposed a maximum likelihood method to estimate the parameters of his model. Another possibility, suggested in the context of AIDS modelling by Ball and O'Neill [20], would be to take $\beta_{x y}=\beta /$ $(x+y), \gamma_{x y}=\gamma$. 
In order to take into account parameter uncertainty, we have adopted a Bayesian decision-theoretic approach. Under this approach it is necessary to choose a distribution for the unknown model parameters. This may be done by referring to previous epidemic data or expert opinion. A review of parameter estimation procedures for epidemic models is given by Becker [21]. In estimating epidemic model parameters, authors have principally considered perfect information in the form of full infection and removal time data. Relatively recently, the more realistic situation where we only have access to imperfect data (usually in the form of removal times or final sizes only) has been considered. Andersson and Britton [22] investigate the estimation of epidemic parameters for both completely and partially observed epidemics. O'Neill and Roberts [23] devised a scheme to estimate the parameters of the general stochastic epidemic model when only removal times (and not infection times) are observed. Within a Bayesian framework, they developed a Markov chain Monte Carlo algorithm to evaluate the joint posterior distribution of the infection and removal rate parameters $\beta, \gamma$, from which it is straightforward to numerically evaluate the posterior distribution of $q=\gamma /(\gamma+\beta)$.

In order to implement our optimal policies, it is necessary to know the state $(X, Y)$ of the population at every time. As noted above, a more realistic situation is that removal times only are observed, so that the value of $X+Y$ is known, but not the values of $X$ and $Y$ separately. The problem of optimal intervention in this situation of incomplete information is the subject of ongoing work.

A further possible extension to our work would be to allow for updating of our uncertainty distributions as the epidemic progresses, to take account of the extra information provided by observation of the infection process. As mentioned above, O'Neill and Roberts [23] show how to update prior distributions for the parameters $\beta, \gamma$ of the general stochastic epidemic model to give a joint posterior distribution for $\beta, \gamma$ at any time during the epidemic. The question of the extent to which adaptive updating of model parameter distributions affects intervention decisions remains an area for further study.

In conclusion, it is clear that real-life public health authorities will not have access to true model parameter values, and thus the hope is that this work may lead to insights of practical value when making control decisions and aid the implementation of an optimal policy.

\section{Acknowledgements}

The authors are grateful to Sean Collins for helpful remarks. The work was carried out while Nathan Green was supported by an EPSRC studentship.

\section{References}

[1] K. Wickwire, Mathematical models for the control of pests and infectious diseases: a survey, Theor. Pop. Biol. 11 (1977) 182.

[2] D. Clancy, Epidemic models, control, in: Peter Armitage, Theodore Colton (Eds.), Encyclopedia of Biostatistics, second ed., vol. 3, John Wiley and Sons, Ltd, 2005, p. 1653.

[3] D.J. Daley, J. Gani, Epidemic Modelling: an Introduction, Cambridge University Press, Cambridge, 1999.

[4] H. Cai, X. Luo, Stochastic control of an epidemic process, Int. J. Syst. Sci. 25 (1994) 821. 
[5] J.A. Bather, Decision theory, Wiley-interscience Series in Systems and Optimization, John Wiley \& Sons, Ltd, 2000.

[6] J.O. Berger, Statistical Decision Theory and Bayesian Analysis, second ed., Springer Series in Statistics, SpringerVerlag, New York, 1985.

[7] N.T.J. Bailey, The Mathematical Theory of Infectious Diseases and its Applications, second ed., Griffin, London, 1975.

[8] A. Abakuks, Some Optimal Isolation and Immunisation Policies for Epidemics, D.Phil. Thesis, University of Sussex, 1972.

[9] A. Abakuks, An optimal isolation policy for an epidemic, J. Appl. Prob. 10 (1973) 247.

[10] A. Abakuks, Optimal immunisation policies for epidemics, Adv. Appl. Prob. 6 (1974) 494.

[11] D. Clancy, Optimal intervention for epidemic models with general infection and removal rate functions, J. Math. Biol. 39 (1999) 309.

[12] E.G. Kyriakidis, Optimal control of two competing diseases or species, Math. Sci. 20 (1995) 56.

[13] E.G. Kyriakidis, Optimal isolation policies for controlling two competing diseases, Math. Sci. 24 (1999) 56.

[14] E.G. Kyriakidis, T.D. Dimitrakos, Optimal control of two competing diseases with state-dependent infection rates, Math. Sci. 27 (2002) 36.

[15] N. Green, Optimal Intervention for Epidemic Models under Uncertainty, Ph.D. Thesis, University of Liverpool, 2005.

[16] J.J. Martin, Bayesian Decision Problems and Markov Chains, Wiley, New York, 1967.

[17] K. Dietz, The estimation of the basic reproduction number for infectious diseases, Stat. Methods Med. Res. 2 (1993) 23.

[18] J.A.P. Heesterbeek, K. Dietz, The concept of R0 in epidemic theory, Stat. Neerl. 50 (1996) 89.

[19] N.C. Severo, Generalisations of some stochastic epidemic models, Math. Biosci. 4 (1969) 395.

[20] F.G. Ball, P.D. O’Neill, A modification of the general stochastic epidemic motivated by AIDS modelling, Adv. Appl. Prob. 25 (1993) 39.

[21] N.G. Becker, Analysis of Infectious Disease Data, Chapman and Hall, London, 1989.

[22] H. Andersson, T. Britton, Stochastic Epidemic Models and Their Statistical Analysis, Springer: Lecture Notes in Statistics, New York, 2000.

[23] P.D. O’Neill, G.O. Roberts, Bayesian inference for partially observed stochastic epidemics, J. R. Statist. Soc. A 162 (Part 1) (1999) 121. 